\title{
Randomized trial of umbilical incisional hernia in high-risk patients: extraction of gallbladder through subxiphoid port vs. umbilical port after laparoscopic cholecystectomy
}

\author{
Min $\mathrm{Li}^{1}$, Baoqiang Cao ${ }^{2}$, Renhua Gong², Dengqun Sun ${ }^{2}$, Peisong Zhang ${ }^{2}$, Xudong Jiang ${ }^{2}$, Yanfei Sheng ${ }^{3}$ \\ ${ }^{1}$ Department of General Surgery, Anhui University of Traditional Chinese Medicine, Hefei, China \\ ${ }^{2}$ Department of General Surgery, Anhui Armed Police General Hospital, Hefei, China \\ ${ }^{3}$ Department of Emergency Department, Anhui Armed Police General Hospital, Hefei, China
}

Videosurgery Miniinv 2018; 13 (3): 342-349

DOI: https://doi.org/10.5114/wiitm.2018.76001

\begin{abstract}
Introduction: Trocar site incisional hernia (TSIH) is one of the most common complications of laparoscopic surgery. Using the umbilical port as a common hole for removing the gallbladder in laparoscopic cholecystectomy is more likely to lead to TSIH than other ports. Thus, extracting the gallbladder through other ports may reduce the incidence of TSIH.

Aim: To ascertain whether extraction of the gallbladder through the subxiphoid port is more beneficial for reducing umbilical incisional hernia than the umbilical port.

Material and methods: From April 2014 to March 2017, a randomized clinical trial was conducted among patients with high risk of incisional hernia and accepted for three-port laparoscopic cholecystectomy (TLC) in our department. 182 patients with indications of cholecystectomy were allocated randomly to group $A$ (subxiphoid port) and group $B$ (umbilical port). Data collection was carried out on operative time, postoperative pain, hospital stay, wound infection and TSIH in the early postoperative course, and at 1, 10, and 24 months after surgery.

Results: The incidence of TSIH in group A was lower than that in group B (4.9\% vs. 14.6\%; odds ratio = 8.02; 95\% Cl: 2.15-47.6; $p$ 0.001). The mean operative time of group $A$ was significantly shorter than that of group $B$ ( $35 \pm 15.16 \mathrm{~min}$ vs. $42 \pm 14.58 \mathrm{~min}, p<0.01$ ). There was no significant difference in wound infection rate between group $A$ and group $B(p=0.068)$. The data of hospital stay $(p=0.428)$ and postoperative pain $(p=0.349)$ of all analyzed patients were similar in the two groups.

Conclusions: Extraction of the gallbladder through the subxiphoid port can reduce umbilical incisional hernia in high-risk patients effectively.
\end{abstract}

Key words: laparoscopic cholecystectomy, subxiphoid port, umbilical port, trocar site incisional hernia.

\section{Introduction}

Laparoscopic surgery has been recognized by many patients for its advantages of small incision, less pain and quick recovery since 1987 [1]. However, reports of complications also increase with the increasing number of laparoscopic operations and changing surgical methods. Among these complications, trocar site incisional hernia $(\mathrm{TSIH})$ is the most common [2, 3]. Several papers agree that the most frequent location of TSIH is the umbilical port and the incidence of this complication is within the range of $0.18 \%$ to $2.8 \%[2,4]$. This outcome may be

Address for correspondence

Baoqiang Cao, Department of General Surgery, Anhui Armed Police General Hospital, Hefei, China, e-mail: caobaoqiang@126.com 
related to the weaker anatomic region of the linea alba, the larger diameter of trocar incision and the extraction of the specimen by frequent fascia dilations $[5,6]$, which also can be seen after laparoscopic cholecystectomy (LC). Furthermore, some publications $[7,8]$ agree that the incidence of this complication has risen to $6.3 \%, 22 \%$ or even higher among high-risk patients [9-11] with comorbidities such as advanced age, diabetes mellitus and obesity. Therefore, preventing TSIH effectively, especially preventing $\mathrm{TSIH}$ in high-risk patients, is of great significance.

Although many studies have shown that the incidence of TSIH can be reduced through methods such as the closure of the fascia if trocar holes $\geq 10 \mathrm{~mm}$ $[12,13]$, the use of prophylactic mesh $[14,15]$ and the use of blunt trocars in trocar holes $[16,17]$, most authors prefer to extract the specimen from the umbilical port, which is the most likely place for TSIH to occur. Thus, to extract the specimen from another feasible and effective port is important. Based on our clinical practice, we believe that the subxiphoid port is feasible and effective.

\section{Aim}

Our study's goal is to evaluate whether the extraction of the gallbladder through the subxiphoid port can control and reduce the incidence of TSIH among high-risk patients without increasing pain, surgical wound infection, operative time and hospital stay.

\section{Material and methods}

\section{Study design}

Our study was approval by the local ethics committee and carried out at the Department of General Surgery, Anhui Armed Police General Hospital, China from April 2014 to March 2017.

One hundred and eighty-two patients who underwent three-port laparoscopic cholecystectomy (TLC) were included in our prospective randomized controlled study to compare the effect of gallbladder extraction through the subxiphoid port or the umbilical port.

We chose high-risk patients as subjects and extended the follow-up to 24 months (March 2015 to March 2017) because the incidence of TSIH might be closer to reality than reported.

\section{Study population}

Our study enrolled patients from 18 years old to 87 years old who suffered from symptomatic gallstones or polyps and satisfied at least one of the high-risk factors for TSIH, such as advanced age (older than 60 years), diabetes mellitus and obesity (body mass index $\geq 30 \mathrm{~kg} / \mathrm{m}^{2}$ ). Exclusion criteria included patients who were converted to laparotomy, had upper-abdominal surgery in the past or were lost to follow-up.

\section{Study procedures}

Surgeons who participated in the operation used standardized procedures. All operations were performed by three experienced surgeons.

\section{Intervention}

Patients who did not have associated allergy history received perioperative systemic antibiotic prophylaxis with cefuroxime (1.5 g IV, Shenzhen Zhijun Pharmaceutical Co., Ltd, Shenzhen, China), and all trocars were inserted through transverse incisions. After three-port laparoscopic cholecystectomy (TLC), all gallbladders were extracted with aseptic gloves to avoid the impacts of ruptured gallbladder and falling stones on incisions.

\section{Group A}

All patients received TLC in the dorsal elevated position and leaning toward the left side at $15^{\circ}$. A 10-mm subumbilical incision for the 10-mm trocar was made by a surgeon who was on the left side of patients. Subsequently, the $10-\mathrm{mm}$ trocar was inserted with a $30^{\circ}$ laparoscope after the implementation of $12 \mathrm{~mm} \mathrm{Hg}$ insufflation of the abdominal cavity. Then, under direct vision, another 10-mm trocar was placed in the subxiphoid incision and a $5-\mathrm{mm}$ trocar was inserted in the right subcostal incision along the midclavicular (the third port). Through the corresponding 5-mm trocar, one grasper was used to retract the gallbladder and expose the Calot triangle. After clear and adequate dissection of the cystic duct, 3-4 endoclips were applied, then the artery and the duct were transected. Afterwards, the gallbladder was stripped away from its bed, put in the sterile gloves and extracted from the subxiphoid port. Our study group adopted continuous 4-0 absorbable sutures (Shanghai Pudong Jinhua Medical 
Products Co., Ltd, Shanghai, China) to sew up subcutaneous tissues (only 2 stitches), and interrupted 2-0 non-absorbable sutures (Ke Hui Medical Equipment Co., Ltd, Sichuan, China) to sew up the skin of the subxiphoid region (only 2 stitches).

\section{Group B (TLC)}

The surgical procedure of group B was similar to that of group A. The only difference was that after removing the gallbladder from the gallbladder bed and put it in a sterile glove, the $30^{\circ}$ laparoscope was removed from the umbilical port and then transferred to the subxiphoid port. Subsequently, under direct vision, the gallbladder was extracted from the umbilical port. Continuous 4-0 absorbable sutures (Shanghai Pudong Jinhua Medical Products Co., Ltd, Shanghai, China) were used to sew up subcutaneous tissues (only 2 stitches), and 2-0 non-absorbable interrupted sutures (Ke Hui Medical Equipment Co., Ltd, Sichuan, China) were used to sew up the skin of the umbilical region.

\section{Randomization method and sample size}

Eligible patients were divided into group $A$ and group B using sealed envelopes which contained random numbers generated by a computer. The randomization was performed 2 days before preparative surgery.

In terms of previous data on TSIH in our center, $4.5 \%$ of patients were without any risk factors, and $8.9 \%$ of patients had high-risk factors. Taking 162 patients as samples, the study used a two-tailed test with a type-l error of $5 \%$ and statistical power of $80 \%$ to calculate a significant sample size. Considering a $10 \%$ dropout rate, the final sample size was 182 with 91 in each group.

\section{Outcome measures}

Surgery time was recorded by a special video during TLC. After TLC, all patients were escorted to sickrooms. The test of postoperative pain was undertaken at 6 h, $24 \mathrm{~h}$, and 3 days after surgery using a visual analog scale (VAS, available though www. rasch.org/rmt/rmt122s.htm). Wound infection was evaluated by a surgeon blinded to the treatment during hospital stay and 30 days after surgery. The primary endpoint of our study was the diagnosis of $\mathrm{TSIH}$. All patients underwent a thorough physical examination to check specifically for signs of a TSIH during the hospital stay. They were given instructions on the clinical signs of a TSIH and were told to contact our department if they found these signs during the follow-up. The diagnosis of TSIH was performed clinically, but if the surgeon suspected it but could not confirm it by physical examination, a computed tomography (CT) scan was carried out.

\section{Statistical analysis}

Our statistical analyses were carried out using SPSS (version 19.0, SPSS Inc). Means and SD were used to describe quantitative variables which followed a normal distribution. Median and range were used to describe quantitative variables that followed a non-Gaussian distribution. Moreover, Student's t-test was used to compare continuous variables and the equivalent nonparametric tests were used when any of these variables did not follow a normal distribution. Qualitative variables were compared by the $\chi^{2}$ test and the differences were quantified by the odds ratio (OR). Logistic regression was applied to perform multivariate analysis and the difference was statistically significant $(p<0.05)$.

\section{Results}

\section{Characteristics of enrolled patients}

In total 194 patients were selected for TLC. Among them, 12 patients were excluded, because 7 patients declined to participate in the study for opposition of their family and so did 5 patients for inconvenience of return visit. Then, 182 patients were randomly assigned to the extraction of the gallbladder through the subxiphoid port (group $A$, $n=91$ ) or the umbilical port (group $\mathrm{B}, n=91$ ). After a 2-year follow-up, the final analysis included 163 patients, among whom 81 patients were in group A (10 patients lost to follow-up or discontinued intervention) and 82 patients were in group $B$ (9 patients lost for the same reasons) (Figure 1).

Characteristics of patients are shown in Table I. The mean age of the two groups was $61.8 \pm 16.5$ years. There were 52 (31.9\%) males and 111 (68.1\%) females showing no difference in body mass index (range: 19.5-48.6 in group A vs. 21.640 in group $B ; 4 \mathrm{~kg} / \mathrm{m}^{2}$ ). In addition, there was no difference in the correlative representation of basic diseases, such as glycuresis, asthma, chronic, and acute cholecystitis. 


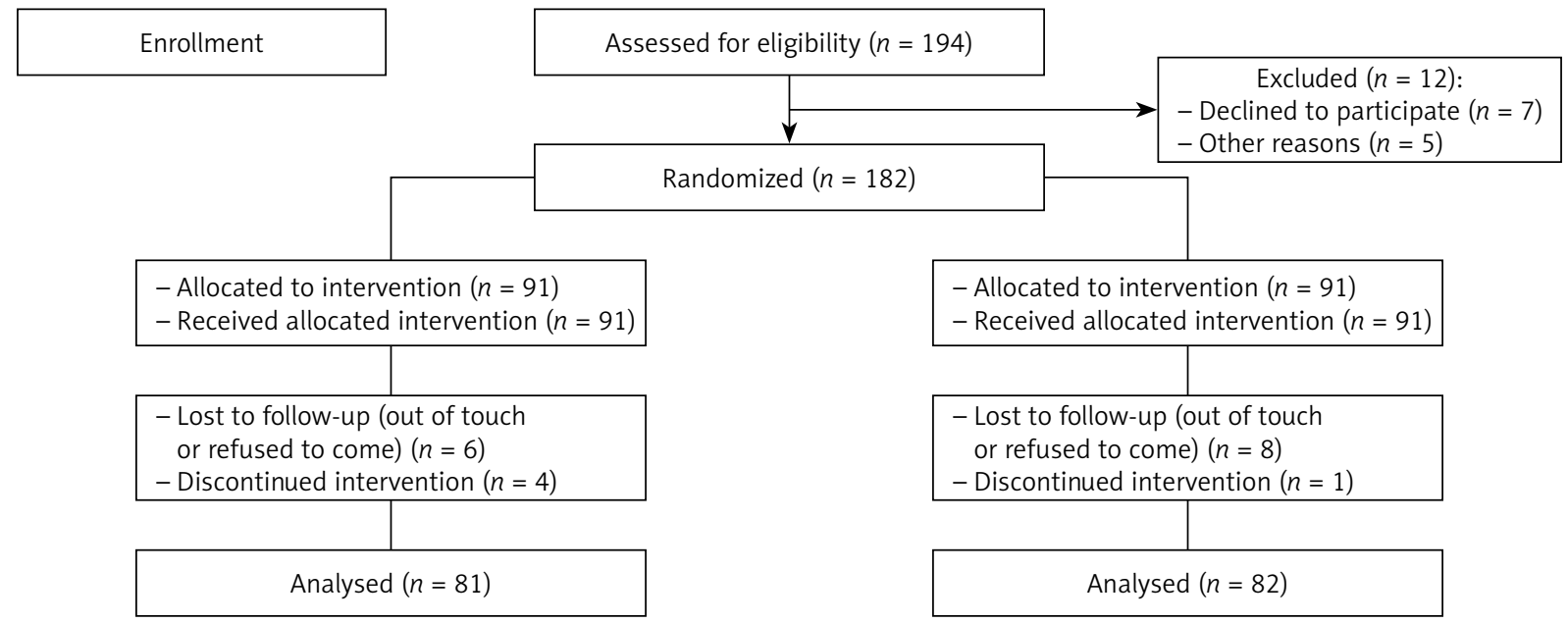

Figure 1. Enrollment - flow diagram

\section{Intraoperative outcome}

The total surgical time of group A was significantly shorter than that of group B ( $35 \pm 15.16$ vs. 42 $\pm 14.58 ; p<0.01)$ and the amount of bleeding was similar between the two groups $(p=0.069)$. The surgery of both groups was completed without conversion to open laparotomy. Also, there did not occur biliary injury or bile leakage (Table II).

\section{Postoperative outcome}

The hospital stay $(p=0.428)$ and the postoperative pain $(p=0.349)$ of all analyzed patients were similar to each other for group A and group B. In addition, the difference in frequency of wound infection between the two groups ( $2.45 \%$ vs. $3.66 \% ; p=0.068)$ was not statistically significant. Moreover, there was no difference for TSIH before discharge $(p=1)$. However, during follow-up, the total incidence of $\mathrm{TSIH}$ was $11.7 \%, 4.9 \%$ in group A and $14.6 \%$ in group B $(\mathrm{OR}=8.02 ; 95 \% \mathrm{Cl}: 2.15-47.6 ; p<0.001)$. The number of these patients needed to treat was 5 (Table III). One hundred and three (63.2\%) patients had 1 risk factor, $36(22.1 \%)$ patients had 2 risk factors, and 22 (13.5\%) patients had 3 risk factors. Only 2 pa-

Table I. Characteristics of patients enrolled in two groups

\begin{tabular}{|lccc|}
\hline Parameter & Group A (subxyphoid) & Group B (umbilical) & $P$-value \\
\hline Age, mean \pm SD [years] & $62.1 \pm 17.1$ & $61.5 \pm 15.9$ & 0.625 \\
\hline Sex, $n$ (\%): & & & 0.614 \\
\hline Male & $25(30.9)$ & $27(32.9)$ & \\
\hline Female & $56(69.1)$ & $55(67.1)$ & 0.824 \\
\hline BMI, mean \pm SD $\left[\mathrm{kg} / \mathrm{m}^{2}\right]$ & $30.5 \pm 6.4$ & $30.3 \pm 6.9$ & 0.756 \\
\hline Glycuresis, $n(\%)$ & $15(18.5)$ & $17(20.7)$ & 0.728 \\
\hline Hypertension, $n(\%)$ & $29(35.8)$ & $31(37.8)$ & 0.701 \\
\hline Hyperlipidemia, $n(\%)$ & $32(39.5)$ & $30(36.6)$ & 0.312 \\
\hline Asthma, $n$ (\%) & $5(6.1)$ & $3(3.7)$ & 0.214 \\
\hline Cholecystolithiasis, $n(\%)$ & $42(51.9)$ & $39(47.6)$ & 0.526 \\
\hline Chronic cholecystitis, $n(\%)$ & $55(67.9)$ & $52(63.4)$ & 0.611 \\
\hline Acute cholecysttitis, $n(\%)$ & $12(14.8)$ & $14(17.0)$ & \\
\hline
\end{tabular}

$B M I-$ body mass index. 
Table II. Intraoperative outcomes associated with two groups

\begin{tabular}{|lccc|}
\hline Parameter & Group A (subxyphoid) & Group B (umbilical) & $P$-value \\
\hline Surgery time, mean \pm SD [min] & $35 \pm 15.16$ & $42 \pm 14.58$ & $<0.01$ \\
\hline Blood loss, median (range) $[\mathrm{ml}]$ & $2(1-10)$ & $2(1-20)$ & 0.069 \\
\hline
\end{tabular}

Table III. Postoperative outcomes associated with two groups

\begin{tabular}{|c|c|c|c|}
\hline Parameter & Group A (subxyphoid) & Group B (umbilical) & $P$-value \\
\hline Pain of VAS, median (range) & $4(1-6)$ & $4(1-7)$ & 0.349 \\
\hline \multicolumn{4}{|l|}{ Wound infection: } \\
\hline Before discharge & 0 & 0 & 1 \\
\hline During follow-up & $2(2.45 \%)$ & $3(3.66 \%)$ & 0.068 \\
\hline \multicolumn{4}{|l|}{ TSIH: } \\
\hline Before discharge & 0 & 0 & 1 \\
\hline During follow-up & $4(4.9 \%)$ & $12(14.6 \%)$ & $<0.001$ \\
\hline
\end{tabular}

VAS - visual analogue scale.

tients had 4 risk factors for TSIH (1.2\%). We found a significant correlation between increased risk for $\mathrm{TSIH}$ and two or more risk factors $(p=0.019$; OR $=$ $4.5 ; 95 \% \mathrm{Cl}: 1.1-9.5)$.

\section{Discussion}

Trocar site incisional hernia is considered the most common complication after laparoscopic surgery $[2,3]$. Since 1995, large published series have shown that the incidence was from $0.18 \%$ to $2.8 \%$ $[13,18-20]$. However, the reported outcomes of these series were lacking a complete or a medium to long-term follow-up. The reason was that patients were always asymptomatic or paid little attention to the observed symptom. Thus, the actual incidence of TSIH might be higher than reported. In our study, the follow-up was permitted by patients and extended to 24 months. All analyzed patients received rigorous screening and periodic review by experienced surgeons. Eventually, the outcome proved that the real situation of $\mathrm{TSIH}$ was higher than expected (11.7\%). We concluded that the incidence of $11.7 \%$ was not only associated with the long-term and effective follow-up, but also with the selection of highrisk patients.

Previous studies revealed that the factors related to the mechanism of TSIH occurrence could be divided into two groups. One was related to patient characteristics and the other was related to surgical techniques and the used materials [14, 21, 22].

For patient characteristics, most published series suggested that patients with obesity (BMI $\geq 30 \mathrm{~kg}$ / $\mathrm{m}^{2}$ ), advanced age (age > 60 years), diabetes mellitus and wound infection were more likely to suffer $\mathrm{TSIH}[15,19,23]$.

The reasons of TSIH occurring in obese patients were their higher intra-abdominal pressure (IAP) and more difficulties in achieving full-thickness closure $[17,24,25]$. In agreement with these studies, we found that it was difficult to close the full thickness of obese patients' substantially thick abdominal wall without enlarging the skin wound through the usual sutural way in our clinical department. (Detailed information can be seen from group A.) The wellknown technical difficulties among obese patients could have contributed to our results [5, 26, 27]. Thus, with the growth of obesity in recent years, it is worthy of further study to solve the problem of incision closure with new preventive measures.

An analysis carried out by Uslu et al. [28] showed that the frequent occurrence of TSIH in older people (age > 60 years) was due to the weaker fascia and abdominal wall structure, which enabled the omentum and the small intestine to break through the confines of these weak areas and protrude outwards.

Furthermore, some authors have stated that there was an obvious relation between diabetes 
mellitus and TSIH. Apart from the influence of diabetes on the healing of tissues, this relation was difficult to explain $[29,30]$.

Surgical site infection (SSI) was considered as a major complication after surgical procedures [31], which contributes to increasing length of hospital stay and healthcare costs. The SSI rate in LC was in the range of $0.3 \%$ to $3.4 \%$ [31-35], and some patients' characteristics were considered as high-risk factors for SSI [36, 37]. In our study, the total incidence of SSI was $3.07 \%$, which was a little outside the range established in the literature. In our opinion, the outcome should be closer to the lowest value in the range of $0.3 \%$ to $3.4 \%$ with the application of antibiotics and the removal of the gallbladder by sterile gloves. Thus, excluding other unrelated factors, we believe that the higher incidence may be associated with the selection of high-risk patients, especially the incomplete wound healing in diabetics during follow-up. During the follow-up, 5 patients developed wound infection (Table III), of whom 4 patients had incomplete wound healing because of hyperglycemia and 1 patient was allergic to the sutures.

For surgical techniques and the used materials, most published series deemed that the most common position for TSIH was the umbilical port because the small intestine and omentum majus might be closer to the umbilical trocar site than other sites [ 20 , 38]. Additionally, the anatomical weakness and the frequent use of a large trocar might increase the risk of TSIH [19]. What is more, some series showed that TSIH could be influenced by repeated stretch, fascia dilation and extension of the incision diameter of the umbilical site for removing large concrements or the swollen gallbladder [19, 25]. In our study, all TSIH appeared at the umbilical port while none appeared at the right subcostal port or subxiphoid port. Also, the only difference between the two groups was the port from which the gallbladder was extracted. After analysis, we surmised that the umbilical site played a more important role than trocar size, especially if associated with other risk factors such as diabetes mellitus, elderly patients, obesity and enlargement of the fascial wound. We insisted that extraction of the gallbladder from the subxiphoid port could avoid unnecessary stretching and dilation of fascia at the umbilical site. Meanwhile, it could also avoid unnecessary extension of the incision diameter for removing large concrements or the swollen gallbladder from the umbilical port.
In terms of studies reviewed, it was clear that the diameter of the trocar hole played an important role in the occurrence of $\mathrm{TSIH}$. Therefore, many authors $[5,12,13]$ have suggested that the fascia should be closed to prevent TSIH when trocar holes $\geq 10 \mathrm{~mm}$. When incisions were $\leq 10 \mathrm{~mm}$, some studies [39-41] suggested closing them when patients had some of the risk factors mentioned before. Although closing the incisions was an effective way to prevent $\mathrm{TSIH}$, current technical difficulty in applying sutures is also serious. Especially in the case of patients with obesity, the overall effectiveness of a simple suture closure was questionable. Therefore, some studies have suggested that both the fascia and all incision levels should be sutured [42, 43]. However, it was difficult to implement this technology without direct vision and extending the incision. Therefore, some authors have proposed the application of prosthetic material when all suture technology could not ensure the effects of wound closure [43, 44]. Armañanzas et al. carried out a comparative study on the superiority of closure by prophylactic mesh placement vs. simple sutures in laparoscopic cholecystectomy and suggested that prophylactic mesh placement could prevent the incisional hernia effectively [14]. In our randomized controlled trial, we did not compare the different suture methods of two groups. In both groups A and group B continuous 4-0 absorbable sutures were used to sew up subcutaneous tissues (only 2 stitches) and interrupted 2-0 non-absorbable sutures were used to sew up the skin of the subxiphoid and umbilical regions (only 2 stitches). The reason why we selected this suture method was because suturing both subcutaneous tissues and skin was not only better than the simple suturing of skin in high-risk parents, but also was well mastered by surgeons in our hospital. This suture method was performed by our experienced doctor Cao in all cases.

The strengths of our study are identifying the relationship between the true incidence of trocar site incisional hernia and long-term, close follow-up. However, our study also has several limitations. One limitation is that the study lacks information on how the fascia and the skin were dilated in the case of extraction of large concrements or a swollen gallbladder. We decided to do carry out additional clinical studies to focus on this issue. Finally, it is also important to study the risk of complications in patients with asymptomatic $\mathrm{TSIH}$. 


\section{Conclusions}

Extraction of the gallbladder through the subxiphoid port can significantly reduce surgical time and the incidence of TSIH in high-risk patients. This method is safe and effective without an increase in hospital stay, postoperative pain or wound infection.

\section{Conflict of interest}

\section{The authors declare no conflict of interest.}

\section{References}

1. Rosen M, Ponsky J. Minimally invasive surgery. Japan J Urol 1992; 33: 823-9.

2. Comajuncosas J, Vallverdú H, Orbeal R, et al. Trocar site incisional hernia in laparoscopic surgery. Cir Esp 2011; 89: 72-6.

3. Moreno-Sanz C, Picazo-Yeste JS, Manzanera-Díaz M, et al. Prevention of trocar site hernias: description of the safe port plug technique and preliminary results. Surg Innov 2008; 15: 100-4.

4. Duca S, Bala O, Al-Hajjar N, et al. Laparoscopic cholecystectomy: incidents and complications. A retrospective analysis of 9542 consecutive laparoscopic operations. HBP 2003; 5: 152-8.

5. Tonouchi H, Ohmori Y, Kobayashi M, et al. Trocar site hernia. Arch Surg 2004; 139: 1248-56.

6. Shaher Z. Port closure techniques. Surg Endosc 2007; 21: 1264-74.

7. Rodríguez JAP, Pera M, Posa LG. Incidence of incisional hernia after open and laparoscopic colorectal cancer resection. Cir Esp 2013;91: 44-9.

8. Höer J, Lawong G, Klinge U, et al. Influencing factors on the causes of incisional hernia. A retrospective study of 2983 laparotomy patients over a period of 10 years. Chirurg 2002; 73: 474-80.

9. Hesselink VJ, Luijendijk RW, de Wilt JH, et al. An evaluation of risk factors in incisional hernia recurrence. Surg Gynecol Obstet 1993; 176: 228-34.

10. Comajuncosas J, Hermoso J, Gris P, et al. Risk factors for umbilical trocar site incisional hernia in laparoscopic cholecystectomy: a prospective 3-year follow-up study. Am I Surg 2014; 207: $1-6$.

11. Wang XC, Zhang D, Yang ZX, et al. Mesh reinforcement for the prevention of incisional hernia formation: a systematic review and meta-analysis of randomized controlled trials. I Surg Res 2017; 209: 17-29.

12. Sanz López R, Martínez Ramos C, Núñez Pena JR, et al. Incisional hernias after laparoscopic vs. open cholecystectomy. Surg Endosc 1999; 13: 922-4.

13. Imme A, Cardi F. Incisional hernia at the trocar site in laparoscopic surgery. Chir Ital 2006; 58: 605-9.

14. Armañanzas L, Ruiz-Tovar J, Arroyo A, et al. Prophylactic mesh vs suture in the closure of the umbilical trocar site after laparoscopic cholecystectomy in high-risk patients for incisional hernia. A randomized clinical trial. J Am Coll Surg 2014; 218: 960-8.

15. Llaguna OH, Avgerinos DV, Nagda P, et al. Does prophylactic biologic mesh placement protect against the development of incisional hernia in high-risk patients? World J Surg 2011; 35: 1651-5.

16. Park MG, Kang J, Kim JY, et al. Trocar site hernia after the use of 12 -mm bladeless trocar in robotic colorectal surgery. Surg Laparosc Endosc Percutan Tech 2012; 22: e34-6.

17. Chiong E, Hegarty PK, Davis JW, et al. Port-site hernias occurring after the use of bladeless radially expanding trocars. Urology 2010; 183: 574-80.

18. Mayol J, Garcia-Aguilar J, Ortiz-Oshiro E, et al. Risks of the minimal access approach for laparoscopic surgery: multivariate analysis of morbidity related to umbilical trocar insertion. World I Surg 1997; 21: 529-33.

19. Coda A, Bossotti M, Ferri F, et al. Incisional hernia and fascial defect following laparoscopic surgery. Surg Laparosc Endosc Percutan Tech 1999; 9: 348-52.

20. Bowrey DJ, Blom D, Crookes PF, et al. Risk factors and the prevalence of trocar site herniation after laparoscopic fundoplication. Surg Endosc 2001; 15: 663-6.

21. Goodenough CJ, Ko TC, Kao LS, et al. Development and validation of a risk stratification score for ventral incisional hernia after abdominal surgery: hernia expectation rates in intra-abdominal surgery (the HERNIA Project). J Am Coll Surg 2015; 220: 405-13.

22. Rogmark P, Petersson U, Bringman S, et al. Quality of life and surgical outcome 1 year after open and laparoscopic incisional hernia repair: PROLOVE: a randomized controlled trial. Ann Surg 2016; 263: 244-50.

23. Elkhadrawy OH, Moussa G, Mansour O, et al. Prophylactic prosthetic reinforcement of midline abdominal incisions in highrisk patients. Hernia 2009; 13: 267-74.

24. Burcharth J, Rosenberg J. Hernias as medical disease. Ugeskrift for Laeger 2008; 170: 3314-8.

25. Hussain A, Mahmood H, Singhal T, et al. Long-term study of port-site incisional hernia after laparoscopic procedures. JSLS 2009; 13: 346-9.

26. Cleveland RD, Laws HL. Incisional closure in morbidly obese patients. Am Surg 1989; 55: 61-3.

27. Eid GM, Collins J. Application of a trocar wound closure system designed for laparoscopic procedures in morbidly obese patients. Obes Surg 2005; 15: 871-3.

28. Uslu HY, Erkek AB, Cakmak A, et al. Trocar site hernia after laparoscopic cholecystectomy. I Laparoendosc Adv Surg Tech A 2007; 17: 600-3.

29. Beltrán MA, Cruces KS. Incisional hernia after McBurney incision: retrospective case-control study of risk factors and surgical treatment. World J Surg 2008; 32: 596-601.

30. Hornby ST, Mcdermott FD, Coleman M, et al. Female gender and diabetes mellitus increase the risk of recurrence after laparoscopic incisional hernia repair. Ann Royal Coll Surg Engl 2015; 97: 115-9.

31. Richards C, Edwards J, Culver D, et al. Does using a laparoscopic approach to cholecystectomy decrease the risk of surgical site infection? Ann Surg 2003; 237: 358-62.

32. Rotermann M. Infection after cholecystectomy, hysterectomy or appendectomy. Health Rep 2004; 15: 11-23.

33. Brandt C, Sohr D, Behnke M, et al. Reduction of surgical site infection rates associated with active surveillance. Infect Control Hosp Epidemiol 2006; 27: 1347-51. 
34. Tucker JJ, Yanagawa F, Grim R, et al. Laparoscopic cholecystectomy is safe but underused in the elderly. Am Surg 2011; 77: 1014-20.

35. Warren DK, Nickel KB, Wallace AE, et al. Risk factors for surgical site infection after cholecystectomy. Open Forum Infect Dis 2017; 4: ofx036. doi: 10.1093/ofid/ofx036.

36. Haridas M, Malangoni MA. Predictive factors for surgical site infection in general surgery. Surgery 2008; 144: 496-503.

37. Drosdeck J, Harzman A, Suzo A, et al. Multivariate analysis of risk factors for surgical site infection after laparoscopic colorectal surgery. Surg Endosc 2013; 27: 4574-80.

38. Duron JJ, Hay JM, Msika S, et al. Prevalence and mechanisms of small intestinal obstruction following laparoscopic abdominal surgery: a retrospective multicenter study. Arch Surg 2000; 135: 208-12.

39. Reardon PR, Preciado A, Scarborough T, et al. Hernia at 5-mm laparoscopic port site presenting as early postoperative small bowel obstruction. J Laparoendosc Adv Surg Tech A 1999; 9 : 523-5.

40. Paya K, Wurm J, Fakhari M, et al. Trocar site hernia as a typical postoperative complications of minimally invasive surgery among preschool children. Surg Endosc 2008; 22: 2724-7.

41. Lee JH, Kim W. Strangulated small bowel hernia through the port site: a case report. World I Gastroenterol 2008; 14: 6881-3.

42. Velasco JM, Vallina VL, Bonomo SR, et al. Post laparoscopic small bowel obstruction: rethinking its management. Surg Endosc 1998; 12: 1043-5.

43. Morrison CP, Wemyss-Holden SA, Iswariah H, et al. Lateral laparoscopic port sites should all be closed: the incisional spigelian hernia. Surg Endosc 2002; 16: 1364.

44. Petrakis I, Sciacca V, Chalkiadakis G, et al. A simple technique for trocar site closure after laparoscopic surgery. Surg Endosc 1999; 13: 1249-51.

Received: 20.12.2017, accepted: 27.03.2018. 